\title{
Correction to: Reopening of Peripheral and Central Arteries and Veins
}

Henri Justino

\section{Correction to:}

G. Butera et al. (eds.), Atlas of Cardiac Catheterization for Congenital Heart Disease, https://doi.org/10.1007/978-3-319-72443-0

This chapter was originally published without the author "Athar M. Qureshi". The author name has been included in the corrected version of the chapter. 